\title{
Broadband light-extraction enhanced by arrays of whispering gallery resonators
}

Zhu, Xiaolong; Ou, Yiyu; Jokubavicius, Valdas; Syvajarvi, Mikael; Hansen, Ole; Ou, Haiyan; Mortensen, N. Asger; Xiao, Sanshui

Published in:

Applied Physics Letters

Link to article, DOI:

$10.1063 / 1.4771124$

Publication date:

2012

Document Version

Publisher's PDF, also known as Version of record

Link back to DTU Orbit

Citation (APA):

Zhu, X., Ou, Y., Jokubavicius, V., Syvajarvi, M., Hansen, O., Ou, H., Mortensen, N. A., \& Xiao, S. (2012). Broadband light-extraction enhanced by arrays of whispering gallery resonators. Applied Physics Letters, 101(24), 241108. https://doi.org/10.1063/1.4771124

\section{General rights}

Copyright and moral rights for the publications made accessible in the public portal are retained by the authors and/or other copyright owners and it is a condition of accessing publications that users recognise and abide by the legal requirements associated with these rights.

- Users may download and print one copy of any publication from the public portal for the purpose of private study or research.

- You may not further distribute the material or use it for any profit-making activity or commercial gain

- You may freely distribute the URL identifying the publication in the public portal 


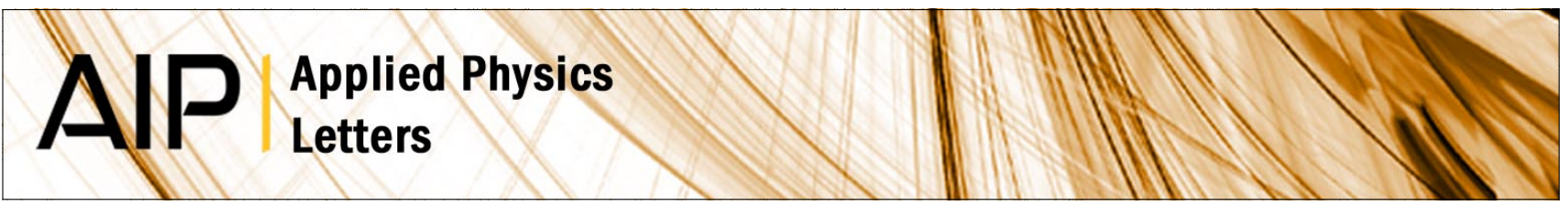

\section{Broadband light-extraction enhanced by arrays of whispering gallery resonators}

Xiaolong Zhu, Yiyu Ou, Valdas Jokubavicius, Mikael Syväjärvi, Ole Hansen et al.

Citation: Appl. Phys. Lett. 101, 241108 (2012); doi: 10.1063/1.4771124

View online: http://dx.doi.org/10.1063/1.4771124

View Table of Contents: http://apl.aip.org/resource/1/APPLAB/v101/i24

Published by the American Institute of Physics.

\section{Related Articles}

High selective two-dimensional Bragg resonators of planar geometry: Theoretical, computational, and experimental study

J. Appl. Phys. 112, 114504 (2012)

Guided-mode quantum efficiency: A novel optoelectronic characterization technique

Rev. Sci. Instrum. 83, 114704 (2012)

An extremely low-noise heralded single-photon source: A breakthrough for quantum technologies

Appl. Phys. Lett. 101, 221112 (2012)

Ultra-thin silicon-on-insulator strip waveguides and mode couplers

Appl. Phys. Lett. 101, 221106 (2012)

Two-dimensional Bessel-like surface plasmon-polariton beams

J. Appl. Phys. 112, 103107 (2012)

\section{Additional information on Appl. Phys. Lett.}

Journal Homepage: http://apl.aip.org/

Journal Information: http://apl.aip.org/about/about_the_journal

Top downloads: http://apl.aip.org/features/most_downloaded

Information for Authors: http://apl.aip.org/authors

\section{ADVERTISEMENT}

\section{AIP Applied Physics Letters}

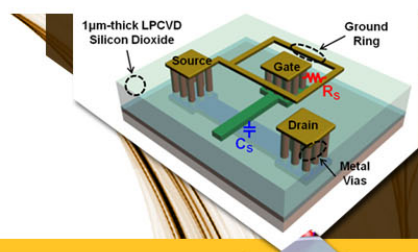

\section{SURFACES AND} INTERFACES

Focusing on physical, chemical, biological structural, optical, magnetic and electrical properties of surfaces and interfaces, and more...

\section{EXPLORE WHAT'S NEW IN APL}

SUBMIT YOUR PAPER NOW!
BNERCY CONVERSION AND STORACE

Focusing on all aspects of static and dynamic energy conversion, energy storage, photovoltaics, solar fuels, batteries, capacitors, thermoelectrics, and more... 


\title{
Broadband light-extraction enhanced by arrays of whispering gallery resonators
}

\author{
Xiaolong Zhu, ${ }^{1, a)}$ Yiyu Ou, ${ }^{1, b)}$ Valdas Jokubavicius, ${ }^{2}$ Mikael Syväjärvi, ${ }^{2}$ Ole Hansen, ${ }^{3}$ \\ Haiyan Ou, ${ }^{1}$ N. Asger Mortensen, ${ }^{1}$ and Sanshui Xiao ${ }^{1, c)}$ \\ ${ }^{1}$ Department of Photonics Engineering, Technical University of Denmark, DK-2800 Kgs. Lyngby, Denmark \\ ${ }^{2}$ Department of Physics, Chemistry and Biology, Linköping University, Linköping SE-58183, Sweden \\ ${ }^{3}$ Department of Micro- and Nano-technology and Center for Individual Nanoparticle Functionality, \\ Technical University of Denmark, DK-2800 Kgs. Lyngby, Denmark
}

(Received 2 July 2012; accepted 27 November 2012; published online 11 December 2012)

\begin{abstract}
We demonstrate a light-extraction approach using a whispering gallery resonators array. The wavelength-scale resonant dielectric nanospheres support whispering gallery modes, which can be coupled with the confined waveguide modes inside the bulk material, thus dramatically improving light extraction. Broadband light-extraction enhancement across the entire visible spectral range is achieved by exciting three low-order and low-quality-factor resonances. As an example, the broadband extraction enhancement of about $50 \%$ is obtained for the emission of fluorescent $\mathrm{SiC}$ at all the tested angles. The experimental results are supported by numerical simulations. Our light-extraction strategy could enable the manufacturing of high-throughput, nondestructive, and affordable optical coating in a variety of optical devices. (C) 2012 American Institute of Physics. [http://dx.doi.org/10.1063/1.4771124]
\end{abstract}

Broadband enhancement of the light-extraction efficiency is especially important for high-performance light-emitting diodes (LEDs). The light extraction efficiency for plain devices is usually low due to the total internal reflection loss arising from the large refractive index contrast between the semiconductor and air. In order to address this, a number of light-extraction schemes have been proposed and demonstrated during the last decades. Traditionally, a single-layer quarter-wavelength thin-film can reduce the light reflection by the destructive interference. Although these coatings can enhance the light extraction for a specific wavelength, the material and thickness of the layer have to be chosen carefully for each wavelength, incident angle, and substrate. Meanwhile, increasing research efforts have been devoted to the light-extraction based on sub-wavelength structures such as photonic crystals, ${ }^{1,2}$ nanorods, ${ }^{3,4}$ and moth-eye ${ }^{5,6}$ structures. The photonic crystal allows for extraction of guided modes within the very thin film LEDs, and the nanorods and motheye structures with high aspect-ratio and subwavelength-scale are efficient to reduce Fresnel reflection with omnidirectional and broad-band behaviors. However, the fabrication methods require complex and expensive processes and the surface damage may degrade the internal quantum efficiency of the optical devices. ${ }^{7}$ Random structures on the surfaces are costeffective, such as using a random patterned silica colloidal mask for plasma etching, ${ }^{8}$ but the high aspect-ratio and subwavelength-scale conditions cannot be simultaneously satisfied, thus making higher light extraction efficiency a challenge.

In this work, we take advantage of whispering gallery (WG) modes for light extraction through the use of an array of wavelength-scale resonant dielectric nanospheres. ${ }^{9}$

\footnotetext{
${ }^{a)}$ Electronic mail: xizhu@fotonik.dtu.dk.

b)Electronic mail: yiyo@fotonik.dtu.dk.

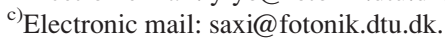

Wavelength-scale dielectric spheres are widely used as the lithographic templates for fabricating various optical structures. $8,10,11$ At the same time, they support low-order WG modes. When the resonant dielectric spheres are in proximity to a high-index active layer of light-emitting devices, a reduction of Fresnel reflection is obtained and an overall suppression in the reflectance spectrum is observed (as in the following discussion). Besides, most of the emitted light propagating as waveguide-like modes inside the high-index material can diffractively couple with the WG modes in the nanospheres, thereby increasing light extraction. The periodically arranged nanospheres array can lead to the coupling of the WG modes, resulting in abundant photonic band-structure with mode splitting and shifting. ${ }^{12-14}$ Donor-acceptor doped fluorescent $\mathrm{SiC}$ is chosen to demonstrate this concept, which is a highly efficient wavelength converter material for white LEDs. ${ }^{15}$ However, the light extraction efficiency of the SiCbased LED is generally low. ${ }^{16,17}$ By simply applying a monolayer array of wavelength-scale dielectric spheres on the plain $\mathrm{SiC}$, an excellent broadband enhancement of light extraction is achieved. We believe that the single-step, nondestructive, and economic optical design can be potentially used for manufacturing more efficient LEDs in industry.

Homoepitaxial layers of $6 \mathrm{H}-\mathrm{SiC}(200 \mu \mathrm{m}$ in thickness, $\mathrm{n}=2.65$ ) with nitrogen $(\mathrm{N})$ and boron (B) dopants were used in this letter, grown by the fast sublimation growth process. ${ }^{18}$ Then, monodispersed suspensions of colloidal polystyrene (PS) nanospheres (600 $\mathrm{nm}$ in diameter, $\mathrm{n}=1.59)$ were coated onto the N-B doped $6 \mathrm{H}-\mathrm{SiC}$ using the Langmuir-Blodgett method. Fig. 1(a) is a schematic illustration of the white LED consisting of a nitride-based near UV LED chip, the $\mathrm{SiC}$ layer for wavelength converting, and a monolayer spheres array. An oblique-view scanning electron microscope (SEM) figure of the $\mathrm{SiC}$ sample with the spheres array is shown in Fig. 1(b). The reflectance spectra for the bare and structured $\mathrm{SiC}$ samples were measured under normal 

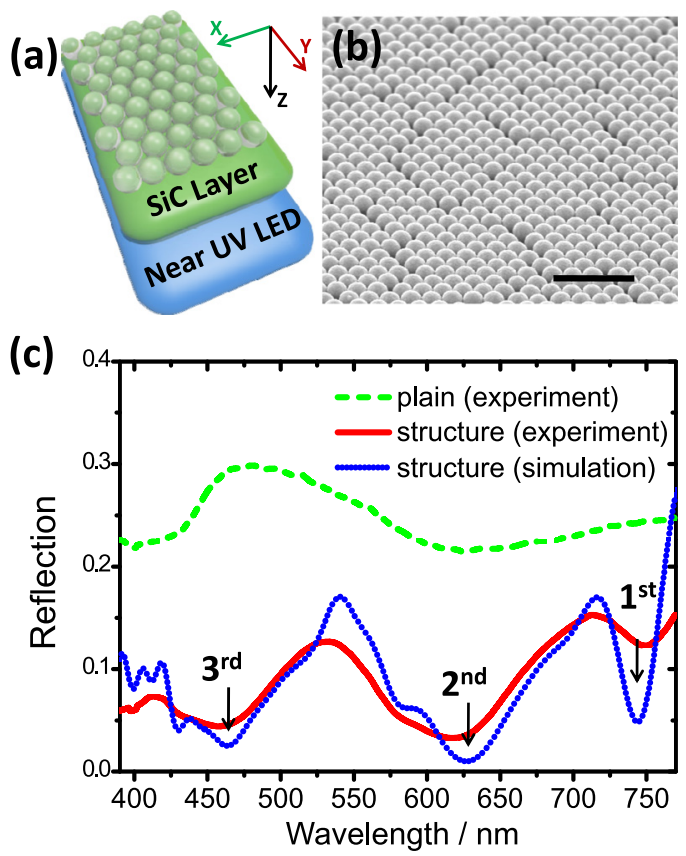

FIG. 1. (a) A schematic illustration of a white LED consisting of a nitridebased near-UV LED chip, a SiC layer for wavelength converting and a monolayer spheres array. (b) An oblique-view SEM figure of the SiC sample with a monolayer array of spheres. Scale bar: $2 \mu \mathrm{m}$. (c) Measured reflectance spectra of the bare $\mathrm{SiC}$ sample (the dashed line) and the one with the spheres array (the solid line), as well as the simulated reflectance (the dotted line).

incidence and the results are shown in Fig. 1(c). The measured wavelength range covers the entire visible spectral range which is typically from 390 to $750 \mathrm{~nm}$. By applying a dielectric resonators array, the surface reflection is effectively suppressed over a broad region, particularly at several specific wavelengths, where a minimum reflectance close to $3 \%$ was observed.

The extraction efficiency $\left(\eta_{\text {ext }}\right)$ can be determined by the fraction of the photons emitted within the extraction cone. For a plain $\mathrm{SiC}$ sample with isotropic emission, it is defined by

$$
\eta_{\text {ext }}=\frac{\int_{0}^{\arcsin \left(n_{\text {air }} / n_{\text {sic }}\right)} T(\theta) 2 \pi \sin (\theta) d \theta}{\int_{0}^{\pi} 2 \pi \sin (\theta) d \theta}
$$

where $T(\theta)$ is the Fresnel transmission factor and can be calculated from the structural parameters. For a plain $\mathrm{SiC}$ sample, the total internal reflection gives an extraction cone with the critical angle $\theta_{c}=\arcsin \left(n_{\text {air }} / n_{S i C}\right) \approx 22^{\circ}$ and the extraction efficiency $\sim 3 \%$. Applying the spheres arrays provides a graded refractive index change for the $\mathrm{SiC} /$ air interface, thus reducing Fresnel reflection. Besides, photons propagating as confined guided modes in the bulk material can be coupled out of the structure with $\theta \geq 22^{\circ}$ when the diffraction condition is satisfied

$$
\left|K_{\|}+G_{m n}\right|<\frac{2 \pi}{\lambda}
$$

Here, $K_{\|}$is the in-plane wavevector, $G_{m n}=2 \pi / \Lambda$ represents the reciprocal lattice vectors of the two-dimensional (2D) lat- tice and $\Lambda=\frac{\sqrt{3}}{2}(m \mathbf{a}+n \mathbf{b})$, where $m$ and $n$ are integers and $\mathbf{a}$ and $\mathbf{b}$ are two basis lattice vectors in the 2D lattice.

To understand the influence of the spheres to the lightextraction efficiency of the SiC based LED structure presented in Fig. 1(a), we performed full-wave simulations based on a finite-integration technique (CST Microwave Studio, CST GmbH, Germany). The simulated reflectance spectrum [the dotted line in Fig. 1(c)] shows a good agreement with the experimental result (the solid line). The reflectance dips appear due to coupling between the spheres array and the $\mathrm{SiC}$ layer. Apart from these strong resonant dips, the splitting of the resonant modes is also observed in Fig. 1(c). Due to the close packing, the cross talk between neighboring spheres causes the spitting of these modes, thus broadening the reflectance dips. This result can also be attributed to the photonic bandstructure of the 2D spheres array which will be discussed later.

Figure 2(a) shows the electric-field patterns of the $E_{y}$ component in the middle of a sphere in the $(x, z)$-plane [Fig. 1(a) at at 745,625 , and $463 \mathrm{~nm}$, as well as the off-resonance one at $540 \mathrm{~nm}$, respectively. Here, the electric field of incident plane waves is along the $x$ direction [Fig. 2(a)]. Two, four, and six lobes are observed inside the sphere for the first three patterns, which are fingerprints of the first-, second-, and third-order resonances of WG modes. The WG modes of the spheres couple with each other and can further couple with the various waveguide modes inside the bulk $\mathrm{SiC}$ layer [Fig. 2(a)]. This coupling can be further proved by the $E_{y}$ electric field in the $(x, y)$-plane of the cross section inside the bulk SiC layer, presented in Fig. 2(b). The second order WG mode is examined in more detail, since its resonant wavelength is located at the wavelength range where the fluorescent $\mathrm{SiC}$ has the emission. In Fig. 2(c), we show the $E_{y}$ electric field component from a cross section in the $\mathrm{SiC}$ layer at $\lambda=625 \mathrm{~nm}$ (left). A strong resonance of the guided mode is excited by the coupling with the WG mode. The offresonance pattern at $\lambda=540 \mathrm{~nm}$ (right) is also shown for comparison. The same contrastive results can also be dig out in the $(x, z)$-plane field patterns in Fig. 2(a). It is worthy to mention that the $E_{y}$ component of the electric field is corresponding to the transverse electric (TE) mode along the $x$ direction. The transverse magnetic (TM) guided mode (not shown) also exists and has the same positive effect for enhancing light-extraction.

There are a large number of optical modes inside the bulk LED structure [Fig. 2(d)]. Each mode has a different electromagnetic field distribution and propagation direction. When the LED active layer is pumped, the generated photons will couple into these optical modes and most of them are confined inside the bulk material. Only the modes propagating within the extraction cone can be extracted for the plain sample. But for the sample with spheres, the confined modes propagating outside the cone can also be extracted by coupling with the WG modes of the spheres, as shown in Fig. 2(d). The average Fresnel transmission factors (T) for samples with and without the spheres $(\lambda=575 \mathrm{~nm}$, the emission peak for doped $\mathrm{SiC}$, is chosen as an example) are numerically calculated and shown in Fig. 2(e) (s-polarization) and 2(f) (p-polarization). Within the extraction cone of plain $\mathrm{SiC}$ sample $\left(\theta \leq 22^{\circ}\right)$, the average transmission factors 
(a)
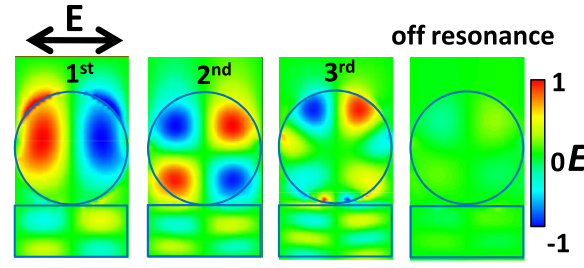

(b)

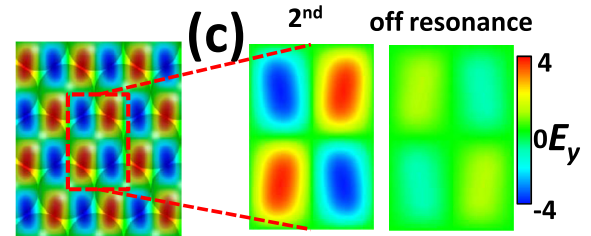

(d)

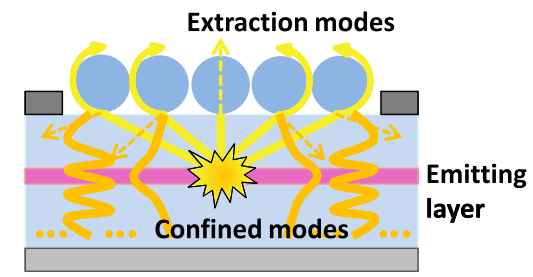

(e)

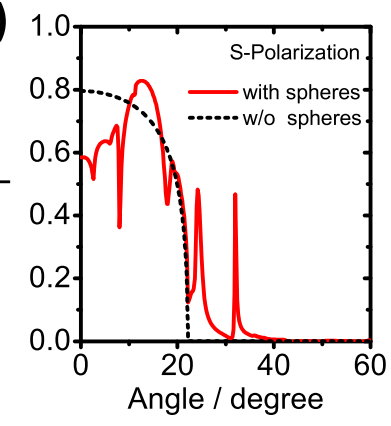

(f)

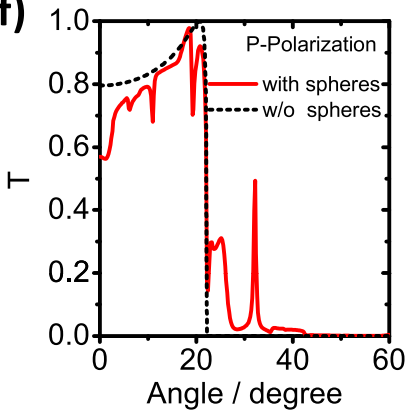

FIG. 2. (a) Electric field distribution of the $E_{y}$ component based on full-wave simulations of electromagnetic waves. Resonance wavelengths are 745,625 , and $463 \mathrm{~nm}$, as well as the off-resonance one at $540 \mathrm{~nm}$, respectively. (b) and (c) $E_{y}$ component of electric field in the $(x, y)$-plane of the cross section inside the bulk $\mathrm{SiC}$ layer. The left pattern in (c) is for $\lambda=625 \mathrm{~nm}$ and the right one is for $\lambda=540 \mathrm{~nm}$, which correspond to the second-order WG mode and the off-resonance frequency, respectively. (d) A schematic illustration of the optical modes confined inside the LED structures and the coupled light-extraction modes are also shown. Fresnel transmission factors for the samples with and without the spheres under (e) s- and (f) p-polarized incidence $(\lambda=575 \mathrm{~nm})$. for samples with and without the spheres are almost the same. Outside the cone $\left(\theta>22^{\circ}\right)$, discrete extraction peaks at specific angles appear for the sample with spheres. We describe these sharp peaks as the characteristic leaky channels provided by the WG resonators. Different from the traditional high-quality-factor (Q) WG resonators, ${ }^{19,20}$ the wavelength-scale resonators presented here possess several advantages for light extraction, such as high energy leakage, low wavelength selectivity, and relatively strong coupling, which are all attributed to the low-Q or broadband characteristic of the low-order WG resonances. ${ }^{21}$ (The calculated Q factors of the first-, second-, and third-order resonances are 30, 6.2, and 5.8, respectively.) Thus, the number of optical modes inside the LED coupling with those WG modes are optimized and the overall light extraction efficiency is increased extremely [Fig. 2(d)].

In order to certificate the above mentioned merits of our structure, we measured the angle-resolved room temperature photoluminescence spectra of the $\mathrm{SiC}$ samples with [Fig. 3(a)] and without [Fig. 3(b)] the spheres array. A $377 \mathrm{~nm}$ diode laser was used as the excitation source which was normal to the sample surface and incident from the backside. The detected emission angle varied from $0^{\circ}$ to $60^{\circ}$. The broadband luminescence of the N-B doped fluorescent $\mathrm{SiC}$ has an emission wavelength range from about 500 to $700 \mathrm{~nm}$. Fig. 3 shows that the $\mathrm{SiC}$ with spheres array illuminates significantly more than the flat sample over the whole emission wavelength range. We can find that the luminescence intensity is enhanced significantly at all the tested angles. This is mainly because the spherical geometry of the WG resonators naturally enables in-coupling at large angles of incidence. In order to verify this, angle-resolved reflectance spectra were measured and shown in Fig. 4(a). The dips are associated with the second-order WG mode in Fig. 1(c). When the angle increases, the modes will further split and shift to short wavelengths, which matches well with the enhancement curves [Fig. 4(b)] evaluated from the data in Fig. 3. The highest extraction enhancement is obtained at the wavelengths where the WG resonances are located and the average enhancement of above $50 \%$ is generally observed in Fig. 4(b).

The angle-dependent modification behavior of the light extraction can be ascribed to the complicated band structure of the 2D photonic-crystal-like close-packed dielectric spheres. The simulation results for the reflectance spectra are
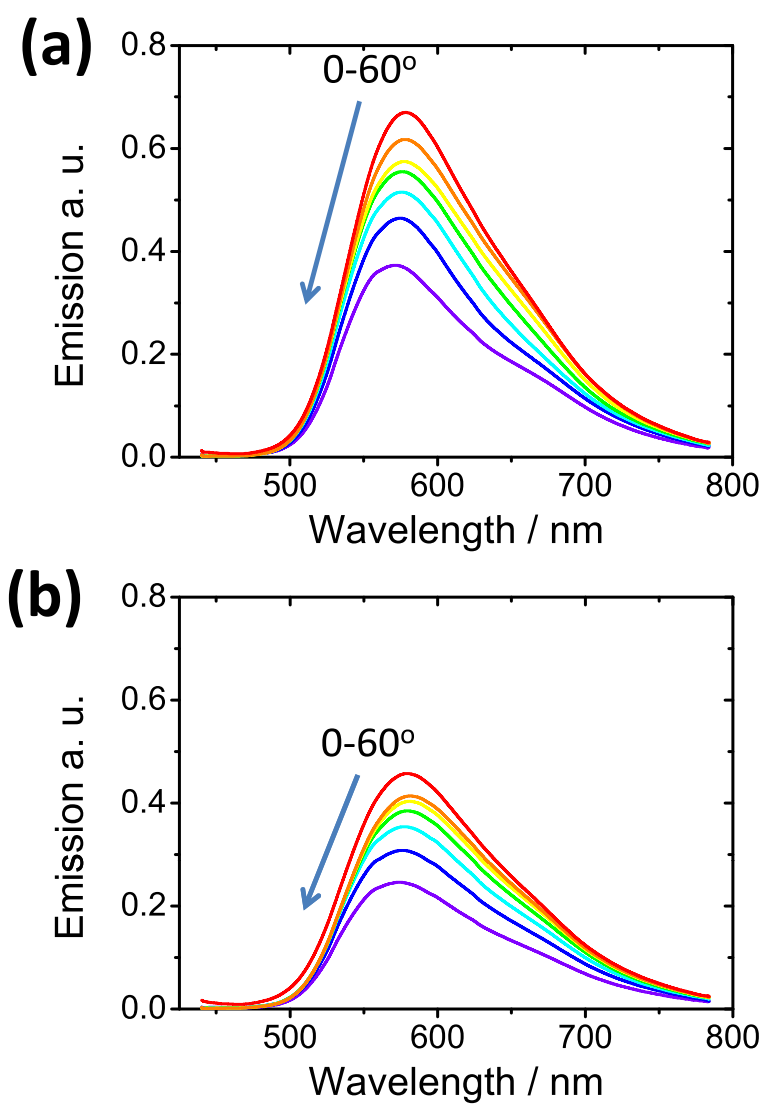

FIG. 3. Angle-resolved photoluminescence spectra from $0^{\circ}, 10^{\circ}, 20^{\circ}, 30^{\circ}$, $40^{\circ}, 50^{\circ}$, to $60^{\circ}$ for the $\mathrm{SiC}$ samples (a) with and (b) without the WG resonators array. 

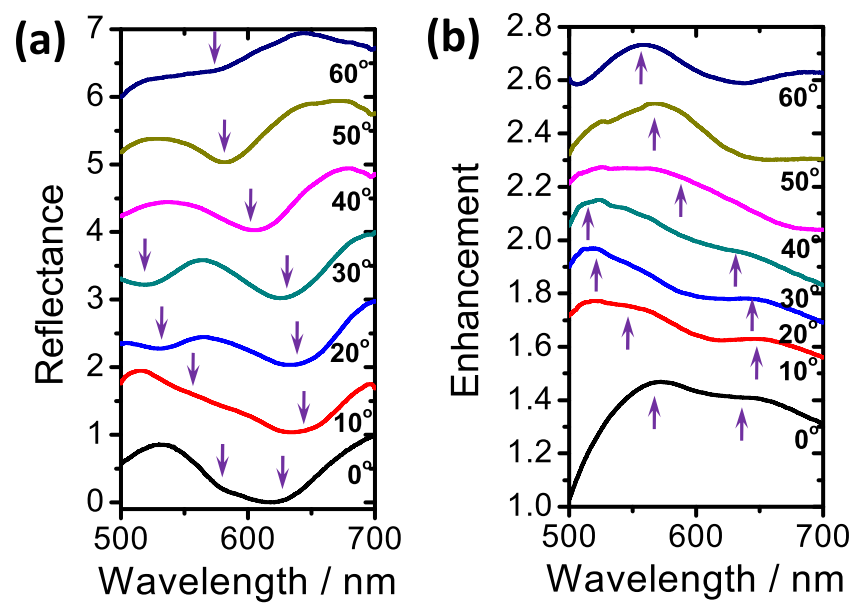

(c)

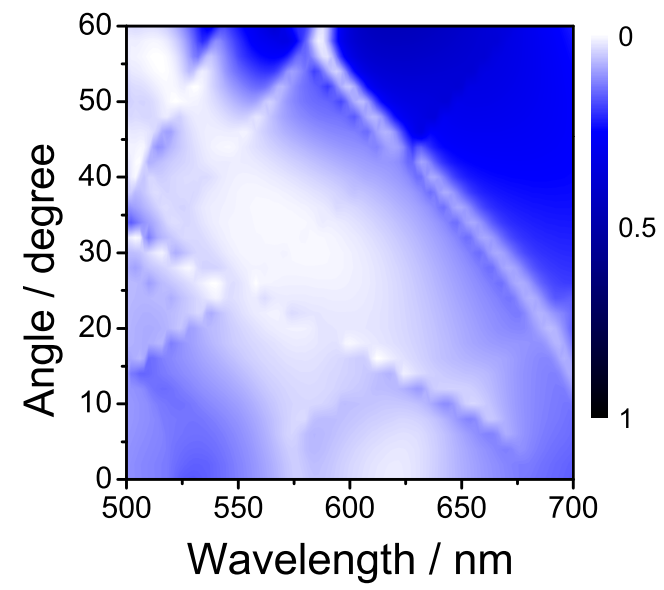

FIG. 4. (a) Angle-resolved reflectance spectra of the $\mathrm{SiC}$ sample with the spheres array. The individual spectra have been normalized and displaced by +1 for clarity. (b) The enhancement curves calculated from the luminescence intensity of the $\mathrm{SiC}$ sample with and without spheres array in Fig. 3. The individual spectra have been displaced by +0.2 for clarity. Arrows in (a) and (b) indicate enhancement peaks shift corresponding to the angledependent resonances of the WG mode. (c) The calculated reflectance spectra for oblique incidence.

shown in Fig. 4(c) for comparison. It is seen that the mode splitting and shifting obtained from the simulation results are in overall agreement with those of the measured results.

In conclusion, we have shown both theoretically and experimentally that a wavelength-scale spheres array deposited on the SiC can strongly modify the light-extraction through coupling multi-order WG modes with the various confined propagating modes inside the bulk SiC. Lightextraction enhancement has been experimentally demonstrated in broadband and across a large range of incident angles, supported by the simulations. The periodically repeated WG modes cover the entire visible spectral range and the spectral positions of these modes can be potentially tuned by varying the sphere diameter and lattice constant. Moreover, by assembling arrays of spheres with different diameters, further enhancement of extraction can be potentially achieved by increasing the number of resonances and by relaxing otherwise strict periodicity. ${ }^{21,22} \mathrm{We}$ believe that the economy and ease of fabrication together with the possibility of tuning their enhancement characteristics will provide us a new route in future efficient LED industry.

This work was supported by the Catalysis for Sustainable Energy Initiative Center, funded by the Danish Ministry of Science, Technology and Innovation, the Danish councils for strategic research funding (No. 09-072118), and the Center for Individual Nanoparticle Functionality (CINF), sponsored by The Danish National Research Foundation.

${ }^{1}$ J. J. Wierer, A. David, and M. M. Megens, Nature Photon. 3, 163 (2009). ${ }^{2}$ S. Fan, P. R. Villeneuve, and J. D. Joannopoulos, Phys. Rev. Lett. 78, 3294 (1977).

${ }^{3}$ S. J. An, J. H. Chae, G.-C. Yi, and G. H. Park, Appl. Phys. Lett. 92, 121108 (2008).

${ }^{4}$ M.-K. Lee, C.-L. Ho, and P.-C. Chen, IEEE Photon. Technol. Lett. 20, 252 (2008).

${ }^{5}$ C.-H. Sun, P. Jiang, and B. Jiang, Appl. Phys. Lett. 92, 061112 (2008).

${ }^{6}$ Y. Ou, V. Jokubavicius, P. Hens, M. Kaiser, P. Wellmann, R. Yakimova, M. Syväjärvi, and H. Ou, Opt. Express 20, 7575 (2012).

${ }^{7}$ S. Noda and M. Fujita, Nature Photon. 3, 129 (2009).

${ }^{8}$ J.-W. Park, J.-H. Park, H.-Y. Koo, S.-I. Na, S.-J. Park, H.-Y. Song, J. W. Kim, W.-C. Kim, and D.-Y. Kim, Jpn. J. Appl. Phys., Part 1 47, 5327 (2008).

${ }^{9}$ J. Grandidier, D. M. Callahan, J. N. Munday, and H. A. Atwater, Adv. Mater. 23, 1272 (2011).

${ }^{10}$ Y. Xia, B. Gates, Y. Yin, and Y. Lu, Adv. Mater. 12, 693 (2000).

${ }^{11}$ X. Zhu, F. Xie, L. Shi, X. Liu, N. A. Mortensen, S. Xiao, J. Zi, and W. Choy, Opt. Lett. 37, 2037 (2012).

${ }^{12}$ H. T. Miyazaki, H. Miyazaki, K. Ohtaka, and T. Sato, J. Appl. Phys. 87, 7152 (2000).

${ }^{13}$ X. Yu, L. Shi, D. Han, J. Zi, and P. V. Braun, Adv. Funct. Mater. 20, 1910 (2010).

${ }^{14}$ L. Shi, H. Yin, X. Zhu, X. Liu, and J. Zi, Appl. Phys. Lett. 97, 251111 (2010).

${ }^{15}$ S. Kamiyama, M. Iwaya, T. Takeuchi, I. Akasaki, M. Syväjärvi, and R. Yakimova, J. Semicond. 32, 013004 (2011).

${ }^{16}$ Y. Ou, V. Jokubavicius, S. Kamiyama, C. Liu, R. W. Berg, M. Linnarsson, R. Yakimova, M. Syväjärvi, and H. Ou, Opt. Mater. Express 1, 1439 (2011).

${ }^{17}$ Y. Ou, D. D. Corell, C. D. Hansen, P. M. Petersen, and H. Ou, Opt. Express 19, A166 (2011).

${ }^{18} \mathrm{M}$. Syväjärvi and R. Yakimova, Encyclopedia-The Comprehensive Semiconductor Science and Technology, (Elsevier, 2011).

${ }^{19}$ C. G. B. Garrett, W. Kaiser, and W. L. Bond, Phys. Rev. 124, 1807 (1961).

${ }^{20}$ V. S. Ilchenko and A. B. Matsko, IEEE J. Sel. Top. Quantum Electron 12, 15 (2006).

${ }^{21}$ Y. Yao, J. Yao, V. K. Narasimhan, Z. Ruan, C. Xie, S. Fan, and Y. Cui, Nat. Commun. 3, 664 (2012).

${ }^{22}$ V. Kitaev and G. A. Ozin, Adv. Mater. 15, 75 (2003). 